\title{
Abundancia, diversidad y huella metabólica de comunidades de nematodos en diferentes zonas de vida en la Región Huetar Norte de Costa Rica
}

\author{
Ingrid Varela Benavides \\ Laboratorio de Nematología, Instituto Tecnológico de Costa Rica, sede San Carlos, Santa Clara, San Carlos; \\ invarela@tec.ac.cr
}

Recibido 23-V-2018. Corregido 07-VIII-2018. Aceptado 04-X-2018.

\begin{abstract}
Abundance, diversity and metabolic footprint of nematode communities in different life zones in the Region Huetar Norte from Costa Rica. Soil biotic communities represent $25 \%$ of the existing global diversity, therefore their study is important for their conservation and sustainable use. Among edaphic biota, nematodes are considered ecologically important as environmental indicators. Tools like the maturity indexes, food web diagnostics and metabolic footprints are used in assessing the ecosystem in relation to the impact contaminants and other stressors, as well as monitoring and measuring changes in the structure and dynamics of the food webs and, more recently, to study the impact of climate factors on the nematode community. Costa Rica is a tropical country with a variety of miroclimates in a small area; this attribute is reflected in the different life zones described by Holdridge for Costa Rica, which differ in their patterns of precipitation, temperature and evapotranspiration. In this research, the diversity of climates was exploited in order to contribute with the knowledge of the nematode communities of several ecosystems within different life zones. For this purpose, samples were taken in several ecosystems located in different life zones in the Region Huetar Norte from Costa Rica. High variation in taxa abundance between different management types within ecosystems was obtained. However, the low availability of replicates for proper statistical analyzes made the mean estimations numerically unprovable. The maturity indexes and the food web diagnosis did not show statistical differences between the studied zones, while, the metabolic footprints were positively correlated to life zones. The metabolic footprint decreased in the different life zones in correspondence with the increase of the average annual temperature reported for each one. The metabolic footprints associated with the decomposition of organic matter (fungivores, bacterivores, and enrichment) had the strongest correlations. The proposition is that the increase in metabolic footprints while the temperature decreases, reflects a change in the dynamics of chemical and biological decomposition of organic matter and in the energy flow in the food networks. This research supports finding in other studies, suggesting that the temperature is a key factor in the species distribution in edaphic ecosystems, and therefore it should be subject to further investigation.
\end{abstract}

Key words: nematode communities; life zones in Costa Rica; microclimates; metabolic footprints maturity indexes.

Varela Benavides, I. (2018). Abundancia, diversidad y huella metabólica de comunidades de nematodos en diferentes zonas de vida en la Región Huetar Norte de Costa Rica. Revista de Biología Tropical, 66(4), 1709-1720.

Las comunidades bióticas que habitan el suelo representan $25 \%$ de la diversidad global existente y sostienen una fracción importante de las funciones de los ecosistemas, constituyendo un valioso recurso cuya importancia fue por mucho tiempo subestimada (Eisenhauer,
Cesarz, Koller, Worm, \& Reich, 2012; Zamioudis \& Pieterse, 2012; Bertsch \& Henríquez, 2015). Por esa razón, investigadores a nivel mundial afirman que es necesario aumentar los estudios sobre los ecosistemas edáficos con el objetivo de conservarlos, gestionar la 
producción y luchar contra el cambio climático (Ferris, Griffiths, Porazinska, Powers, Wang, \& Tenuta, 2012; Eisenhauer et al., 2012; Mueller et al., 2016; Looby \& Treseder, 2018).

Entre la biota edáfica, los nematodos son un grupo muy diverso y cosmopolita que ha sido estudiado por su importancia ecológica, económica, y por ser eficientes indicadores ambientales (Culman et al., 2010; SánchezMoreno \& Talavera, 2013). Cada taxón del filo Nematoda responde de forma diferente a los cambios en el ambiente, por lo que el análisis de la composición de la comunidad nematológica permite evaluar la condición del ecosistema con relación al impacto de contaminantes y otros factores estresantes, así como monitorear los cambios en la estructura y funcionamiento de las redes tróficas del suelo (Sánchez-Moreno \& Talavera, 2013).

Por otro lado, como los nematodos del suelo están presentes en muchos niveles tróficos, inciden en el flujo de nutrientes y energía, con la consecuente influencia en la producción y secuestro de carbono, en la movilización de minerales y en la regulación de las poblaciones microbianas (Ferris, 2010; Ferris et al., 2012; Gingold, Moens, \& Rocha-Olivares, 2013).

Con el fin de simplificar la información que brinda el análisis de las comunidades de nematodos se utilizan índices que permiten resumir los datos en pocas variables. Los índices específicos para este grupo fueron propuestos inicialmente por Bongers (1990), quien desarrolló el índice de madurez, el cual está basado en las diferentes estrategias de sobrevivencia de las familias de nematodos. Posteriormente, se desarrollaron otras herramientas derivadas del mismo: el $\sum$ índice de madurez (Bongers \& Bongers, 1998), el índice de madurez 2-5 (Neher \& Olson, 1999), los índices de la red trófica, (Ferris, Bongers, \& de Goede, 2001) y las huellas metabólicas (Ferris, 2010).

Respecto al estudio del efecto de factores climáticos en las comunidades nematológicas, especialmente en aquellas investigaciones enmarcadas en el área del cambio climático, éstas sugieren que el efecto observado depende del ecosistema bajo análisis. En estudios realizados en la Antártida se determinó que el aumento de la temperatura provoca un aumento en la abundancia, la riqueza de especies y la complejidad de las redes tróficas, además de favorecer la presencia de nuevas especies (Nielsen, Wall, Adams, \& Virginia, 2011). Mientras que en un clima tropical en Ecuador se encontró que la temperatura y el tipo de suelo no influyeron en la diversidad, si bien sí se observó un cambio en la proporción de grupos tróficos dominantes. La disminución de la temperatura favoreció a los nematodos fungívoros sobre los herbívoros en diferentes altitudes, y los autores relacionaron este cambio a la disponibilidad de recursos (Traunspurger, Reiff, Krashevska, Majdi, \& Scheu, 2017).

Los últimos estudios, que incluyen el análisis de huellas metabólicas, consideran que un aumento en la temperatura reducirá la huella metabólica de los nematodos, es decir la participación de los nematodos en el flujo de la energía en las redes tróficas (Bhusal, Tsiafouli, \& Sgardelis, 2015; Kergunteuil, Campos-Herrera, Sánchez-Moreno, Vittoz, \& Rasmann., 2016).

En Costa Rica algunos estudios realizados en plantaciones de café y banano han validado la utilidad de los nematodos como indicadores de la calidad de los suelos costarricenses, y su relación con el tipo de manejo agronómico (Salguero-Londoño, 2006; Peraza, 2010; Rodríguez, Muñóz, \& Pocasangre, 2011). Ninguno de estos estudios ha explorado el efecto de los factores climáticos en las comunidades nematológicas del suelo.

Por otro lado, Costa Rica es un país tropical donde se pueden encontrar gran variedad de microclimas en un área pequeña, esta característica se ve reflejada en las diferentes zonas de vida descritas por Holdridge (1967) para el territorio nacional, las cuales difieren en sus patrones de precipitación, temperatura y evapotranspiración.

En este trabajo se estudiaron las comunidades de nematodos en diversos ecosistemas ubicados en distintas zonas de vida, como una contribución para entender el efecto de los factores climáticos en las comunidades de 
nematodos y su participación en el flujo de la energía en las redes tróficas.

\section{MATERIALES Y MÉTODOS}

Localización del estudio y muestreo: Las comunidades nematológicas de cuatro diferentes zonas de vida (ZV) en la Región Huetar Norte de Costa Rica fueron estudiadas. Las ZV incluidas en el estudio fueron: bosque húmedo montano bajo (HMB), bosque pluvial premontano (BPP), bosque húmedo premontano (HP) $\mathrm{y}$ bosque muy húmedo tropical (MHT). Su descripción, así como la ubicación y características de los lugares muestreados de detalla en el cuadro 1.

Los muestreos se realizaron entre julio 2015 y junio 2016, en ecosistemas naturales y productivos. En cada ecosistema se definió un área de $50 \mathrm{~m}^{2}$ (7 x $7 \mathrm{~m}$ aproximadamente) $\mathrm{y}$ en ella se realizaron 50 perforaciones equidistantes entre sí, a una profundidad de $15 \mathrm{~cm}$ y con un barreno oakfield de $2 \mathrm{~cm}$ de diámetro. Las muestras fueron trasladadas en cajas de aislamiento térmico hasta el Laboratorio de Nematología del Instituto Tecnológico de Costa Rica en Santa Clara de San Carlos y ahí se almacenaron por no más de tres días a $4{ }^{\circ} \mathrm{C}$ hasta la extracción de los nematodos.

\section{Extracción e identificación de nemato-}

dos: Cada muestra de suelo fue homogenizada y cuarteada hasta obtener cinco submuestras de $100 \mathrm{~g}$, las cuales fueron procesadas por medio de un elutriador de Oostenbrink (van Bezooijen, 2006). La separación final de los nematodos de las partículas de suelo se realizó mediante cribas de extracción con doble filtro de algodón. La suspensión resultante con nematodos fue recogida 24 y 48 horas después, fue vertida en una cámara de conteo, y la totalidad de los individuos presentes fue contada con la ayuda de un microscopio invertido Olympus CKX41 con el fin de obtener la abundancia total de nematodos en cada sistema. Seguidamente, cada muestra fue fijada en su totalidad con formalina caliente al $4 \%$, y transferida a glicerina pura usando el método modificado de Seinhorst (1959).

Para la identificación de los nematodos cada muestra fue homogenizada antes de ser dispersada en una cámara de conteo cuadriculada, y los primeros 100 individuos en la cámara fueron recuperados para ser montados en láminas permanentes. Seguidamente fueron identificados a nivel de familia o género utilizando un microscopio compuesto Olympus BX53 con contraste interferencial Nomarski hasta una magnificación de 400X. La abundancia de cada taxón fue expresada como número de nematodos en $100 \mathrm{~g}$ de suelo

Índices de la comunidad de nematodos: Cada grupo taxonómico fue asignado a un grupo trófico según Yeates, Bongers, de Goede, Freckman, \& Georgieva (1993), y a un grupo cp (colonizadores-persistentes) según Bongers (1990) y Bongers \& Bongers (1998). Los grupos cp representan diferentes estrategias de sobrevivencia de los nematodos, a las familias de nematodos colonizadores se les asigna el número 1 en la escala $\mathrm{cp}$, mientras que a los nematodos persistentes un 5 en la misma escala. Los nematodos con características intermedias se les asigna un 2, 3 ó 4, dependiendo de si su estrategia de sobrevivencia se acerca más a la de colonizadores o persistentes. Los nematodos en el extremo inferior de la escala (colonizadores), se consideran oportunistas de ambientes enriquecidos, y por lo tanto indican disponibilidad de nutrientes. Los nematodos en el extremo superior de la escala son persistentes, e indican estabilidad del ecosistema y redes tróficas complejas (Ferris \& Bongers, 2009).

Se calcularon los índices de madurez, como indicadores del estado de sucesión biológica del suelo. Los índices calculados fueron: el índice de madurez, que es el valor ponderado de la distribución de frecuencias de los valores cp en una muestra dada (Bongers, 1990), el índice de fitoparásitos (Bongers, 1990), que se calcula únicamente con las familias de nematodos fitoparásitos, el $\sum$ índice de madurez (Yeates, 1994), en el que se incluyen las familias de fitófagos al realizar los cálculos, el índice de 


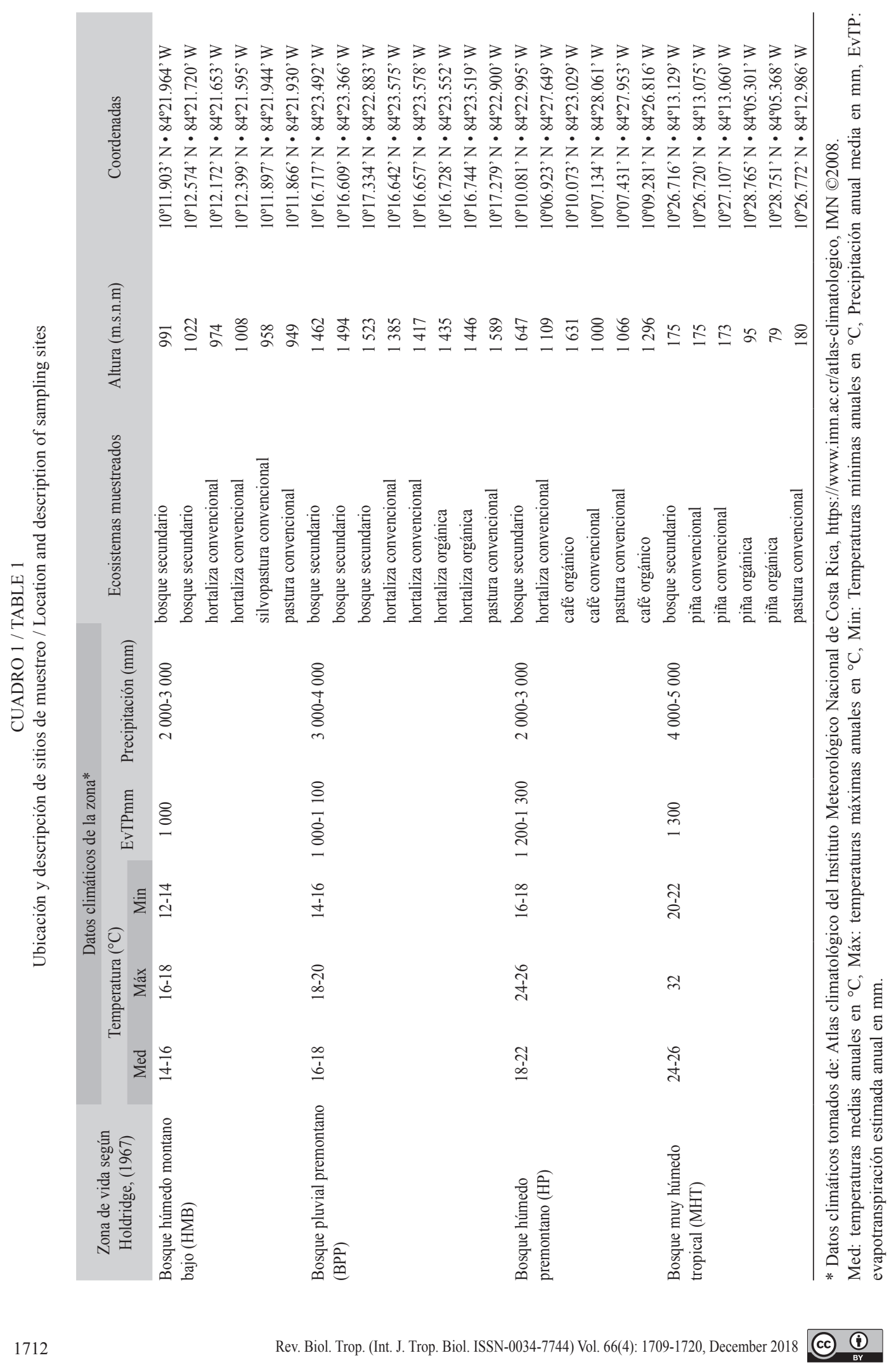


madurez 2-5 (Neher \& Olson, 1999) que excluye los nematodos del grupo cp 1, los cuales han sido llamados por varios autores "oportunistas de ambientes enriquecidos" distinguiéndolos del grupo cp 2 u "oportunistas generales".

Además se calcularon los índices de la red trófica, es decir, el índice de estructura y el índice de enriquecimiento (Ferris et al., 2001), que combinan la escala cp con los grupos tróficos reconocidos entre los nematodos (bacterívoros, fungívoros, herbívoros, omnívoros y depredadores), para establecer los gremios funcionales - los nematodos Ba1 serían colonizadores bacteriófagos, los nematodos Ful serían colonizadores fungívoros y así sucesivamente. Los índices de la red trófica permiten evaluar la complejidad de la misma y el tipo de descomposición de la materia orgánica que predomina en el ecosistema del suelo.

Por último, se calcularon las huellas metabólicas (Ferris, 2010), que tienen en cuenta además de los gremios funcionales, la biomasa de los nematodos en el suelo, la cual se estima para cada género según su tamaño, y se utiliza para evaluar el carbono medio consumido por cada nematodo a lo largo de su ciclo de vida. La huella metabólica puede calcularse como huella metabólica total o por grupo funcional. Las huellas de herbívoros, bacterívoros y fungívoros, indican el canal respectivo por el que ingresa el carbono. La huella de enriquecimiento: es la huella metabólica de aquellos nematodos que responden de manera más rápida al enriquecimiento, y la de estructura es la huella metabólica de los nematodos en los niveles más altos de las redes tróficas.

Todos los índices fueron calculados con ayuda de la herramienta NINJA: Nematode indicator joint analysis (Sieriebriennikov, Ferris \& de Goede, 2014).

Por último, se estimó la diversidad de nematodos a través del índice de diversidad Shannon-Wiener (Shannon \& Weaver, 1949) para cada una de las ZV.

Las diferencias estadísticas entre las abundancias de las diferentes familias en cada ZV y ecosistema fueron comprobadas mediante pruebas de Kruskal-Wallis.
Para determinar diferencias estadísticas entre los índices calculados en las diferentes ZV y ecosistemas se realizaron ANOVAs y pruebas de Tukey. Los índices fueron previamente transformados con logaritmo natural para reducir el efecto de la varianza según recomienda Ferris (2010).

También se estimó la correlación entre los índices calculados para las ZV, la temperatura media y media en cada una mediante el coeficiente Rho de Spearman ( $\rho$ ), que se utiliza como una medida de asociación de datos ordinales.

Los análisis se realizaron con el software estadístico SPSS statistical software (SPSS, Inc., Chicago, IL, USA), las diferencias con un nivel de probabilidad menor a $0.05(\mathrm{P}<0.05)$ fueron consideradas significativas.

\section{RESULTADOS}

Abundancia y composición de la comunidad: En términos de composición de la comunidad no se determinó ninguna tendencia y no se encontraron diferencias significativas respecto a la abundancia de las familias y géneros estudiados entre los diferentes ecosistemas.

Por otro lado, y omitiendo aquellos grupos tróficos poco frecuentes (abundancia media menor que 1), la abundancia de algunas familias fue estadísticamente diferente entre ZV. Individuos de Setinernematidae fueron encontrados únicamente en los HMB y BPP. También las abundancias medias de Aporcelaimidae, Tylenchidae y Qudsianematidae fueron mayores en el HMB $(P<0.05)$, y la abundancia de Dorylaimidae fue significativamente menor en el MHT $(\mathrm{P}=0.02)$. La abundancia promedio de individuos en cada $\mathrm{ZV}$ se presenta en el cuadro 2 .

Por otro lado, la biomasa total de nematodos presentó diferencias, y se correlacionó significativamente con la temperatura media de las ZV $(\rho=0.814, P<0.01)$. Según se observa en el cuadro 2 , la biomasa de nematodos es mayor en el HMB, que presenta temperaturas más bajas y tiende a disminuir en correspondencia 


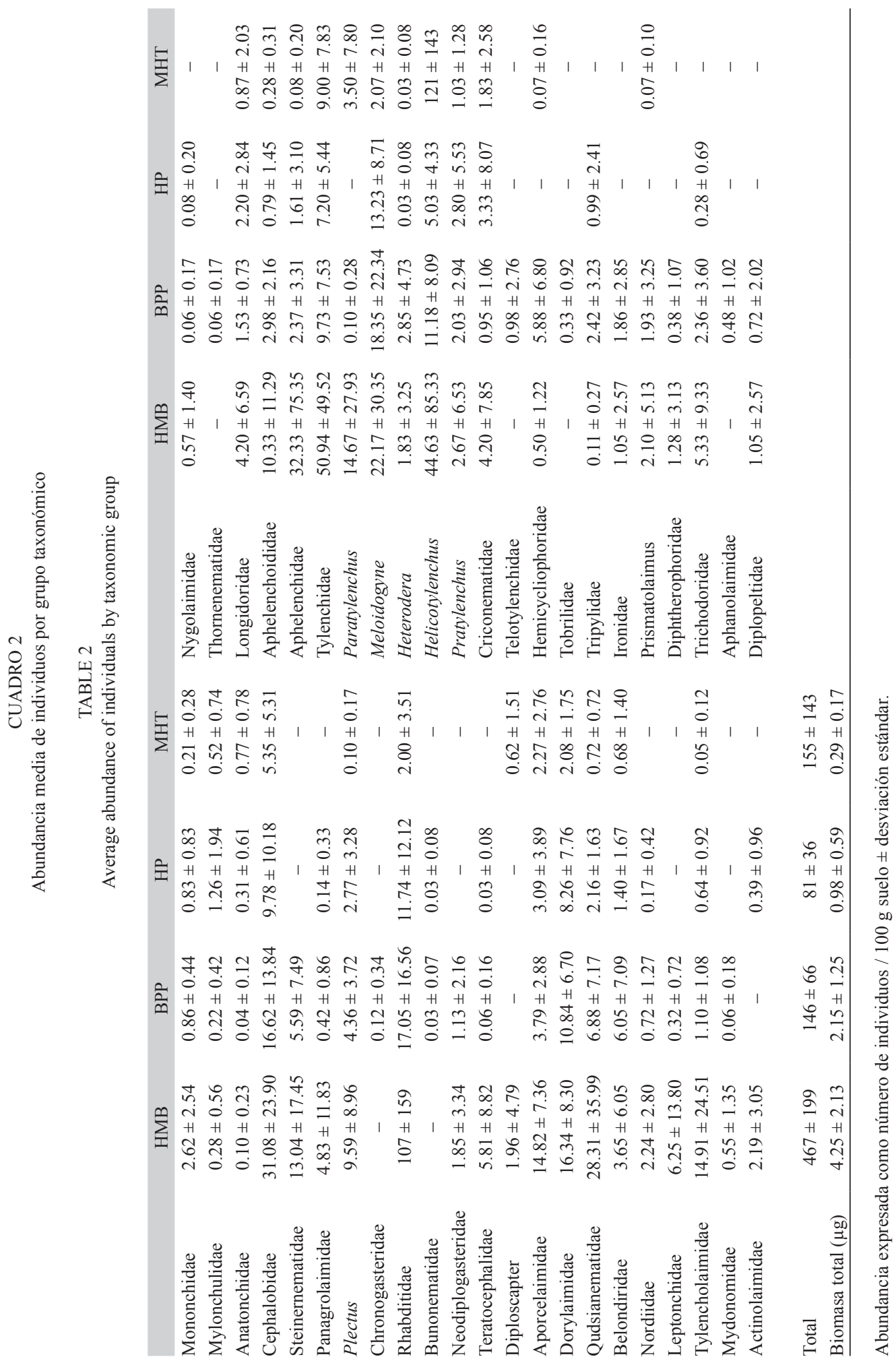


con el gradiente de temperatura que represen$\tan$ las diferentes $\mathrm{ZV}$.

\section{Índices de la comunidad de nematodos:} No se encontraron diferencias significativas entre los índices de madurez, diversidad, ni los de la red trófica en las diferentes ZV (Cuadro 3), y no se obtuvo correlación significativa entre el valor del índice de madurez y la ZV o el tipo de ecosistema estudiado.

Por el contrario, las huellas metabólicas calculadas para cada ZV sí presentaron diferencias significativas (Cuadro 3), y una correlación con la temperatura media en cada ZV mayor de $0.74(\mathrm{P}<0.01)$ en casi todos los casos. Las huellas metabólicas de herbívoros y depredadores fueron la excepción y se obtuvo una correlación de $\rho=0.3$ y $0.25(\mathrm{P}>0.05)$ respectivamente.

La relación entre las huellas metabólicas y el gradiente de temperatura representado por las diferentes $\mathrm{ZV}$ se puede observar en la figura 1 y en la figura 2 . A pesar de la variación en los datos, debido a la diversidad de ecosistemas incluidos en el análisis, se observó una clara correlación entre las huellas metabólicas calculadas y la temperatura media anual en las ZV.

Las mayores correlaciones se obtuvieron con los valores de las huellas metabólicas de bacterívoros $(\rho=0.88 ; \mathrm{P}<0.01)$ y fungívoros $(\rho=0.88 ; \mathrm{P}<0.01)$, estos valores fueron significativamente mayores en el HMB y al igual que la biomasa disminuyeron progresivamente en las ZV con mayor temperatura media anual (Fig. 2).

\section{DISCUSIÓN}

$\mathrm{Al}$ analizar los resultados obtenidos se debe tomar en cuenta la variedad de ecosistemas

CUADRO 3

Valores promedio de índices de diversidad, de madurez, de la red trófica y huellas metabólicas

TABLE 3

Average values of diversity, maturity indexes, trophic network indexes and metabolic footprints

\begin{tabular}{llcccc} 
& Índice & HMB & BPP & HP & MHT \\
Índices de & Índice de madurez & $2.6 \pm 0.99$ & $2.67 \pm 0.39$ & $2.65 \pm 0.8$ & $2.85 \pm 0.73$ \\
madurez & Índice de madurez 2-5 & $3.24 \pm 0.60$ & $3.25 \pm 0.41$ & $3.41 \pm 0.60$ & $3.46 \pm 0.47$ \\
& $\Sigma$ índice de madurez & $2.56 \pm 0.74$ & $2.76 \pm 0.25$ & $2.74 \pm 0.43$ & $2.75 \pm 0.32$ \\
& Índice de fitonematodos & $2.61 \pm 0.27$ & $2.88 \pm 0.20$ & $2.92 \pm 0.21$ & $2.81 \pm 0.24$ \\
Índices de la red & Canal & $11.48 \pm 6.44$ & $5.23 \pm 4.43$ & $8.41 \pm 9.04$ & $40.83 \pm 54.02$ \\
trófica & Basal & $7.63 \pm 5.52$ & $10.39 \pm 9.33$ & $10.01 \pm 5.65$ & $10.21 \pm 8.58$ \\
& Enriquecimiento & $77.84 \pm 11.86$ & $76.68 \pm 17.45$ & $57.19 \pm 36.04$ & $45.09 \pm 46.09$ \\
& Estructura & $79.87 \pm 22.18$ & $84.14 \pm 12.85$ & $86.18 \pm 9.36$ & $87.29 \pm 11.07$ \\
Huellas & Enriquecimiento & $446.94 \pm 302.03 \mathrm{a}$ & $144.71 \pm 149.29 \mathrm{~b}$ & $18.12 \pm 18.18 \mathrm{bc}$ & $3.13 \pm 5.26 \mathrm{c}$ \\
metabólicas & Estructura & $134.97 \pm 67.75 \mathrm{a}$ & $52.58 \pm 23.15 \mathrm{ab}$ & $34.15 \pm 26.44 \mathrm{bc}$ & $16.99 \pm 15.84 \mathrm{c}$ \\
& Herbívoros & $181.89 \pm 216.99$ & $151.52 \pm 171.65$ & $96.34 \pm 60.68$ & $33.29 \pm 22.82$ \\
& Fungívoros & $9.35 \pm 10.64 \mathrm{a}$ & $0.92 \pm 0.66 \mathrm{ab}$ & $0.38 \pm 0.48 \mathrm{bc}$ & $0.04 \pm 0.06 \mathrm{c}$ \\
& Bacterívoros & $450.42 \pm 307.52 \mathrm{a}$ & $148.75 \pm 149.98 \mathrm{a}$ & $20.51 \pm 17.83 \mathrm{~b}$ & $4.04 \pm 4.66 \mathrm{~b}$ \\
& Depredadores & $4.83 \pm 5.17$ & $5.31 \pm 4.93$ & $2.48 \pm 2.75$ & $1.54 \pm 1.18$ \\
\hline Índice de & Omnívoros & $125.99 \pm 59.85 \mathrm{a}$ & $46.91 \pm 23.2 \mathrm{ab}$ & $31.57 \pm 23.9 \mathrm{bc}$ & $15.43 \pm 15.04 \mathrm{c}$ \\
diversidad & Shannon-Wiener & $0.831 \pm 0.59$ & $0.775 \pm 0.32$ & $0.791 \pm 0.40$ & $1.252 \pm 0.51$ \\
\hline
\end{tabular}

Índices expresados como media \pm desviación estándar.

Las casillas sombreadas y seguidas de una letra minúscula indican valores estadísticamente diferentes $(\mathrm{P}<0.01)$ para el valor del índice en las zonas de vida. Las diferencias entre las medias que comparten una letra no son estadísticamente significativas. 


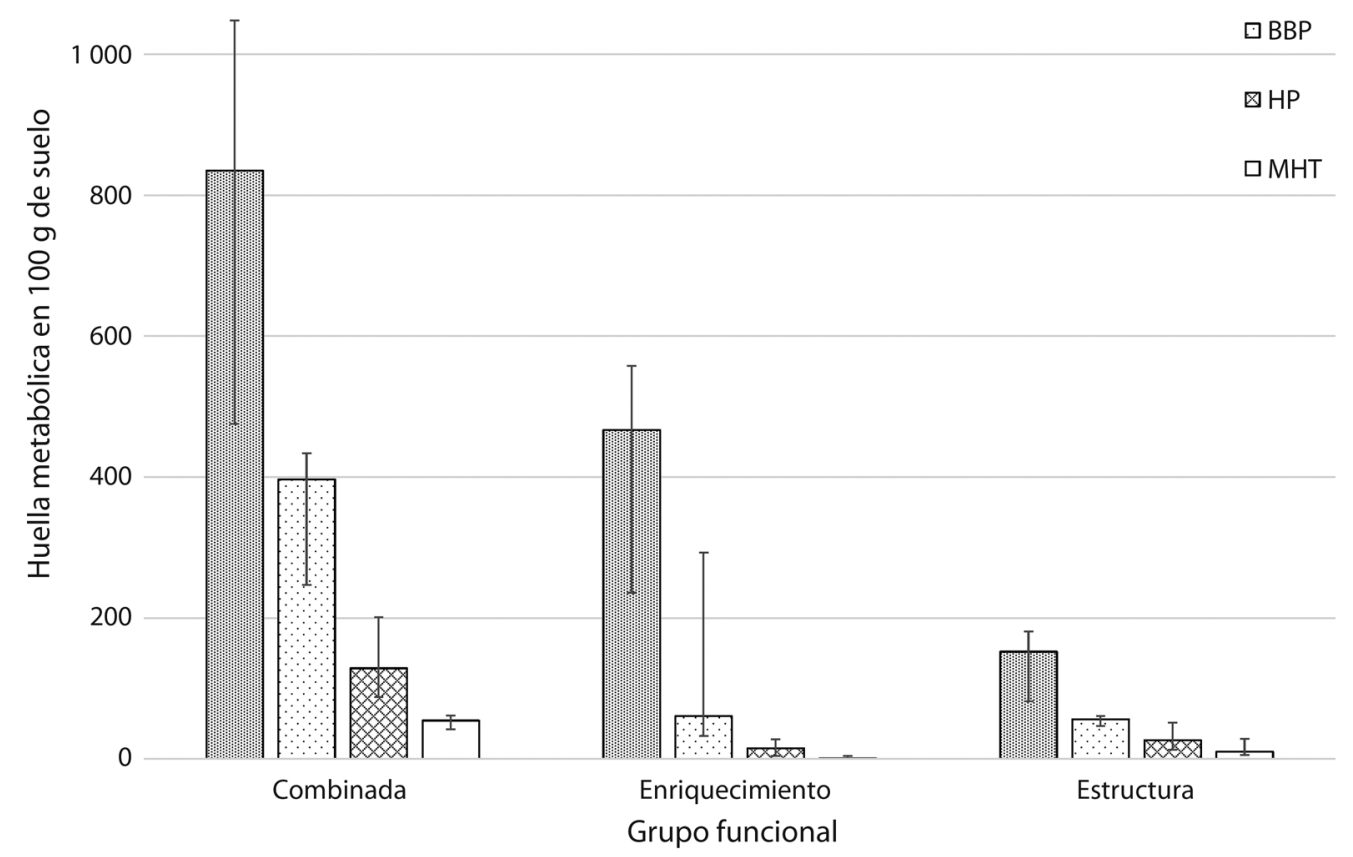

Fig. 1. Distribución de valores de las huellas metabólicas combinadas, de enriquecimiento y de estructura, calculadas para cada zona de vida, las cajas representan los valores de la mediana y las prolongaciones los percentiles 25 y 75 .

Fig. 1. Distribution of the values of the composite footprint, enrichment footprint and structure footprint, calculated for each life zone. The boxes represent the values of the median and the whiskers the 25 th and 75 th percentiles.

incluidos en este estudio, situación que se ve reflejada en los altos valores de las medidas de dispersión calculadas, pero que a la vez permite validar los resultados en un contexto en el que median muchos factores.

La alta variabilidad en los datos fue, probablemente, uno de los factores por los que no fue posible establecer diferencias estadísticamente significativas entre los diferentes ecosistemas respecto a las variables calculadas, por lo que se recomienda aumentar el número de repeticiones en futuras investigaciones. Aunque también es importante considerar que existen otros estudios en los que no se encontró diferencias entre ecosistemas con diferentes grados de manejo (Bloemers, Hodda, Lambshead, Lawton, \& Wanless, 1997; Neher, 1999), lo que es un factor para tomar en cuenta en el uso de los nematodos como indicadores.

Por otro lado, estos resultados reafirman el planteamiento de Ferris (2010), que propone que los índices de madurez y de la red trófica tienen una función descriptiva, y no evalúan la participación de los nematodos en el flujo de la energía, que son los recursos que determinan el tamaño y la actividad de la red trófica. La validación que con el tiempo se haga de esta herramienta podría ayudar a comprender de mejor manera las interacciones de las poblaciones edáficas.

Los resultados obtenidos en este estudio coinciden, parcialmente, con los obtenidos por otros estudios similares (Bhusal et al., 2015, Kergunteuil et al., 2016; Traunspurger et al., 


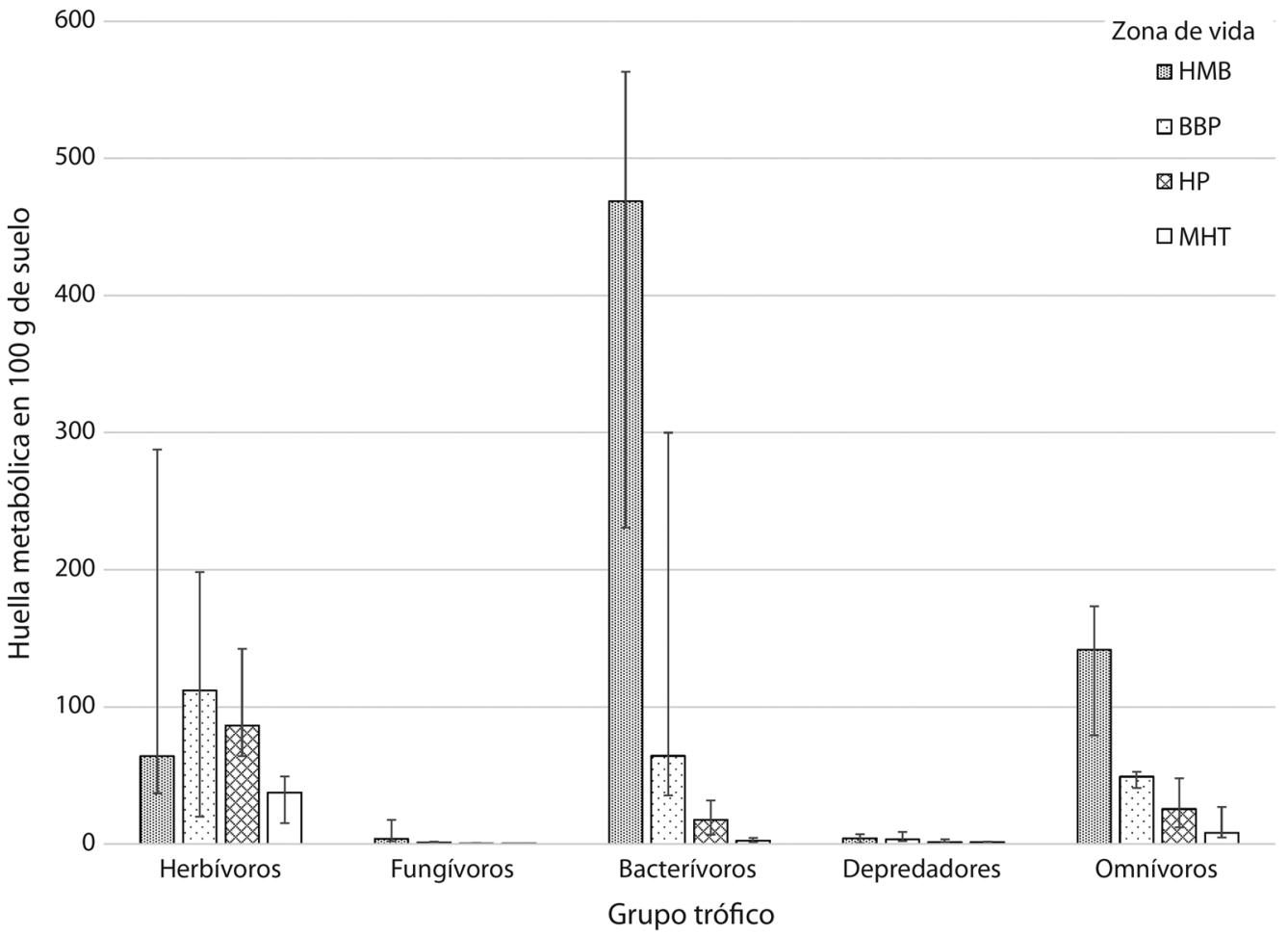

Fig. 2. Distribución de valores de las huellas metabólicas por grupo trófico calculadas para cada zona de vida, las cajas representan los valores de la mediana y las prolongaciones los percentiles 25 y 75 .

Fig. 2. Distribution of the values of the metabolic footprints for each trophic group calculated for each life zone. The boxes represent the values of the median and the whiskers the 25 th and 75 th percentiles.

2017), que apuntan a que el papel que juegan los nematodos en la descomposición de la materia orgánica y las redes tróficas podría ser superior en lugares ubicados a mayor altura, y que la temperatura es uno de los factores que podrían explicar esta relación.

Sin embargo, los trabajos mencionados argumentan que los cambios observados en las huellas metabólicas pueden deberse, en parte, al tipo de vegetación presente en cada microclima y a la cantidad de raíces en cada uno, mientras que en este caso no se logró establecer ninguna correlación entre los diferentes ecosistemas estudiados en cada $\mathrm{ZV}$, los cuales difieren en el tipo de vegetación presente y el manejo, y los índices calculados para la comunidad.
En este caso, la diferencia fue observada en la biomasa de la comunidad nematológica, lo que sugiere que los individuos de las especies presentes en lugares donde la temperatura media anual es menor son de mayor tamaño, y por lo tanto tienen mayor importancia relativa en la red trófica. También se subraya que familias que agrupan especies consideradas grandes, Aporcelaimidae, Qudsianematidae y Dorylaimidae, fueron más abundantes en las ZV con menor temperatura.

El aumento de las huellas metabólicas conforme disminuye la temperatura, evidencia un cambio en la dinámica de la descomposición química y biológica de la materia orgánica y en el flujo de energía en la red trófica que debería ser motivo de estudio. La influencia de la 
temperatura en los procesos enzimáticos, en la descomposición química de la materia orgánica y en la absorción y excreción de los minerales ya se ha estudiado (Davidson \& Janssens, 2006), y podría explicar la composición de las comunidades biológicas en ecosistemas edáficos ubicados en diferentes microclimas.

Otro resultado que apoya la afirmación anterior es que las huellas metabólicas que se correlacionan más fuertemente con la temperatura media en las ZV fueron las que están directamente relacionadas con la descomposición de la materia orgánica del suelo (fungívoros, bacterívoros y la huella del enriquecimiento), mientras que las huellas de los herbívoros y depredadores no se correlacionaron con los sitos estudiados.

Otros autores (Gillingham, Palmer, Huntley, Kunin, Chipperfield, \& Thomas, 2012; Suggitt et al., 2011), ya han mencionado que la temperatura podría influir más directamente en la distribución de las especies que las características del hábitat, lo que tendría importantes implicaciones en las políticas de mitigación del cambio climático y en la conservación que deben ser tomadas en cuenta.

Declaración de ética: los autores declaran que todos están de acuerdo con esta publicación y que han hecho aportes que justifican su autoría; que no hay conflicto de interés de cualquier tipo; y que han cumplido con todos los requisitos y procedimientos éticos y legales pertinentes. El documento firmado se encuentra en los archivos de la revista.

\section{AGRADECIMIENTOS}

A la Vicerrectoría de Investigación del Instituto Tecnológico de Costa Rica, por el financiamiento brindado para la ejecución de este proyecto. A Reyes Peña Santiago por su conocimiento y capacitación en la identificación de familias de dorylaimidos.

\section{RESUMEN}

El estudio de las comunidades bióticas que habitan el suelo y que representan un $25 \%$ de la diversidad existente, es importante para su conservación y aprovechamiento sostenible. Entre la biota edáfica los nematodos se consideran de importancia ecológica como indicadores ambientales. Herramientas como los índices de madurez, los índices de la red trófica y las huellas metabólicas, basadas en la comunidad de nematodos, son utilizadas para evaluar la condición del ecosistema con relación al impacto de contaminantes y otros factores estresantes en los ecosistemas. Los cambios en la estructura y funcionamiento de las redes tróficas del suelo y más recientemente el efecto de los factores climáticos, también tienen impacto en la comunidad nematológica. Costa Rica es un país tropical donde se pueden encontrar gran variedad de microclimas en un área pequeña, esta característica se ve reflejada en las diferentes zonas de vida descritas por Holdridge para el territorio nacional, las cuales difieren en sus patrones de precipitación, temperatura y evapotranspiración. En esta investigación, se aprovechó la diversidad de climas para contribuir con el conocimiento de las comunidades de nematodos de varios ecosistemas en diferentes zonas de vida. Para esto se recolectaron muestras en distintas zonas de vida en la Región Huetar Norte de Costa Rica. Los nematodos presentes en las muestras fueron extraídos e identificados a nivel de familia o género, y con los datos obtenidos se calcularon índices de diversidad, de madurez, de la red trófica y huellas metabólicas. Se obtuvo una gran variación en la abundancia de taxa entre los diferentes tipos de manejo dentro de los ecosistemas; sin embargo, la baja disponibilidad de repeticiones para analizar estadísticamente de manera precisa, hizo que las estimaciones de una media fueran indemostrables numéricamente. No fue posible establecer diferencias significativas entre los ecosistemas con diferentes tipos de manejo respecto a las variables calculadas, lo que se atribuye a la variabilidad de los datos. En cuanto a las zonas de vida, los índices de madurez y de la red trófica no mostraron diferencias entre las mismas, mientras que las huellas metabólicas, así como la biomasa de nematodos se correlacionaron positivamente con éstas. En el bosque húmedo montano bajo, la zona con menor temperatura media anual, la huella metabólica fue mayor, luego la huella metabólica disminuyó en las diferentes zonas de vida en correspondencia con el aumento de la temperatura media anual reportada para cada una. Las huellas metabólicas relacionadas con la descomposición de la materia orgánica del suelo (fungívoros, bacterívoros y enriquecimiento) manifestaron correlaciones altamente significativas. Se plantea que el aumento de las huellas metabólicas conforme disminuye la temperatura evidencia un cambio en la dinámica de la descomposición química 
y biológica de la materia orgánica del suelo y en el flujo de energía de la red trófica. En otros estudios también se ha concluido que la temperatura es un factor determinante en la distribución de las especies en ecosistemas edáficos, y por lo tanto debería ser objeto de mayor investigación.

Palabras clave: comunidades de nematodos; zonas de vida; microclimas; huellas metabólicas; índices de madurez.

\section{REFERENCIAS}

Bertsch, F., \& Henríquez, C. (2015). 2015: El Año Internacional de los Suelos. Agronomía Costarricense, 39(3), 149-155.

Bhusal, D. R., Tsiafouli, M. A., \& Sgardelis, S. P. (2015). Temperature-based bioclimatic parameters can predict nematode metabolic footprints. Oecologia, 179(1), 187-199.

Bloemers, G. F., Hodda, M., Lambshead, P. J. D., Lawton, J. H., \& Wanless, F. R. (1997). The effects of forest disturbance on diversity of tropical soil nematodes. Oecologia, 111, 575-582.

Bongers, T. (1990). The maturity index: an ecological measure of environmental disturbance based on nematode species composition. Oecología, 83, 14-19.

Bongers, T., \& Bongers, M. (1998). Functional diversity of nematodes. Applied Soil Ecology, 10(3), 239-251.

Culman, S. W., Young-Mathews, A., Hollander, A. D., Ferris, H., Sánchez-Moreno, S., O'Geen, A. T., \& Jackson, L. E. (2010). Biodiversity is associated with indicators of soil ecosystem functions over a landscape gradient of agricultural intensification. Landscape Ecology, 25(9), 1333-1348.

Davidson, E. A., \& Janssens, I. A. (2006). Temperature sensitivity of soil carbon decomposition and feedbacks to climate change. Nature, 440(7081), 165-173.

Eisenhauer, N., Cesarz, S., Koller, R., Worm, K., \& Reich, P. B. (2012). Global change belowground: impacts of elevated $\mathrm{CO}_{2}$, nitrogen, and summer drought on soil food webs and biodiversity. Global Change Biology, $18(2), 435-447$.

Ferris, H. (2010). Form and function: metabolic footprints of nematodes in the soil food web. European Journal of Soil Biology, 46(2), 97-104.

Ferris, H., Bongers, T., \& de Goede, R. G. M. (2001). A framework for soil food web diagnostics: extension of the nematode faunal analysis concept. Applied Soil Ecology, 18(1), 13-29.

Ferris, H., \& Bongers, T. (2009). Indices developed specifically for analysis of nematode assemblages. En M. J. Wilson, \& T. Kakouli-Duarte (Eds.), Nematodes as environmental indicators (pp. 124-145). Wallingford, UK: CAB International.

Ferris, H., Griffiths, B. S., Porazinska, D. L., Powers, T. O., Wang, K. H., \& Tenuta, M. (2012). Reflections on plant and soil nematode ecology: past, present and future. Journal of Nematology, 44(2), 115-126.

Gillingham, P. K., Palmer, S. C., Huntley, B., Kunin, W. E., Chipperfield, J. D., \& Thomas, C. D. (2012). The relative importance of climate and habitat in determining the distributions of species at different spatial scales: a case study with ground beetles in Great Britain. Ecography, 35(9), 831-838.

Gingold, R., Moens, T., \& Rocha-Olivares, A. (2013). Assessing the response of nematode communities to climate change-driven warming: a microcosm experiment. PLoS One, 8(6), e66653.

Holdridge, L. R. (1967). Life zone ecology. San José, Costa Rica: Centro Científico Tropical.

Kergunteuil, A., Campos-Herrera, R., Sánchez-Moreno, S., Vittoz, P., \& Rasmann, S. (2016). The abundance, diversity, and metabolic footprint of soil nematodes is highest in high elevation alpine grasslands. Frontiers in Ecology and Evolution, 4, 1-12.

Looby, C. I., \& Treseder, K. K. (2018). Shifts in soil fungi and extracellular enzyme activity with simulated climate change in a tropical montane cloud forest. Soil Biology and Biochemistry, 117, 87-96.

Mueller, K. E., Blumenthal, D. M., Carrillo, Y., Cesarz, S., Ciobanu, M., Hines, J., Pabst, S., Pendall, E., Milano, C., Wall, D. H., \& Eisenhauer, N. (2016). Elevated $\mathrm{CO}_{2}$ and warming shift the functional composition of soil nematode communities in a semiarid grassland. Soil Biology and Biochemistry, 103, 46-51.

Neher, D. A. (1999). Nematode communities in organically and conventionally managed agricultural soils. Journal of. Nematology, 31, 142-154.

Neher, D. A., \& Olson, R. K. (1999). Nematode communities in soils of four farm cropping management systems. Pedobiología, 43, 430-438.

Nielsen, U. N., Wall, D. H., Adams, B. J., \& Virginia, R. A. (2011). Antarctic nematode communities: observed and predicted responses to climate change. Polar Biology, 34(11), 1701-1711.

Peraza, W. (2010). Nematofauna asociada a cultivo de café (Coffea arabica) orgánico y convencional en Aserrí, Costa Rica. Ingenierías \& Amazonia, 3(2), 105-112.

Rodríguez, A., Muñoz, Y. E., \& Pocasangre, L. E. (2011). Evaluación de nematodos de vida libre como indicadores de calidad y salud de suelos en tres sistemas de producción de banano. Tierra Tropical, 8(1), 115-125. 
Salguero-Londoño, B. M. (2006). Caracterización de nematodos de vida libre como bioindicadores de calidad y salud de suelos bananeros en Costa Rica (Tesis de Maestría). Centro Agronómico Tropical de Investigación y Enseñanza, Turrialba, Costa Rica.

Sánchez-Moreno, S., \& Talavera, M. (2013). Los nematodos como indicadores ambientales en agroecosistemas. Ecosistemas, 22(1), 50-55.

Seinhorst, J. W. (1959). A rapid method for the transfer of nematodes from fixative to anhydrous glycerine. Nematologica, 4, 67-69.

Shannon, C. E., \& Weaver, W. (1949). The mathematical theory of communication. Urbana, IL: University of Illinois Press.

Sieriebriennikov, B., Ferris, H., \& de Goede, R. G. M. (2014). NINJA: An automated calculation system for nematode-based biological monitoring. European Journal of Soil Biology, 61, 90-93.

Suggitt, A. J., Gillingham, P. K., Hill, J. K., Huntley, B., Kunin, W. E., Roy, D. B., \& Thomas, C. D. (2011).
Habitat microclimates drive fine-scale variation in extreme temperatures. Oikos, 120(1), 1-8.

Traunspurger, W., Reiff, N., Krashevska, V., Majdi, N., \& Scheu, S. (2017). Diversity and distribution of soil micro-invertebrates across an altitudinal gradient in a tropical montane rainforest of Ecuador, with focus on free-living nematodes. Pedobiologia, 62, 28-35.

van Bezooijen, J. (2006). Methods and techniques for nematology. Wageningen, The Netherlands: Wageningen University Press.

Yeates, G. W. (1994). Modification and qualification of the nematode maturity index. Pedobiología, 38, 97-101.

Yeates, G. W., Bongers, T., de Goede, R. G. M., Freckman, D. W., \& Georgieva, S. S. (1993). Feeding habits in soil nematode families and genera - an outline for soil ecologists. Journal of Nematology, 25, 315-331.

Zamioudis, C., \& Pieterse, C. M. (2012). Modulation of host immunity by beneficial microbes. Molecular Plant-Microbe Interactions, 25(2), 139-150. 